\title{
An Authoring Tool for Generating Context Awareness Mobile Game Based Learning
}

\author{
https://doi.org/10.3991/ijet.v17i02.25943 \\ Lamyae Bennis $^{1}(\llbracket)$, Khalid Kandali ${ }^{2}$, Hamid Bennis ${ }^{2}$ \\ ${ }^{1}$ Faculty of Sciences of Meknes, Moulay Ismail University, Meknes, Morocco \\ ${ }^{2}$ Graduate School of Technology, Moulay Ismail University, Meknes, Morocco \\ lamyaebennis@gmail.com
}

\begin{abstract}
Game-Based Learning (GBL) has become extensively used in Technology-Enhanced Learning (TEL) as a substitute for traditional education methods. Moreover, research has been done in this field proves that it's very interesting for Learning Game (LG) designers to integrate the latest technologies in order to augment GBL efficiency and to reach more learners. On the other hand, context awareness and adaptation are a crucial aspect in mobile gamebased learning. It is thus necessary to place at the disposal of the learner player an adaptive mobile game based learning according to the context. However, the contextualisation in the training is not easy to reach. The diversity of mobile technologies and dynamics in mobile environments complicate the process of contextualisation. Furthermore, migration and implementation of these novel technologies can be very pricey and require more informatics knowledge and time, which can lead to a huge loss. Thus, the main purpose of this article is to present the proposed e-Adventure architecture using the ZeroCouplage multiplatform Framework that was introduced to solve this problem. We code once and the context awareness for mobile game-based learning is automatically generated.
\end{abstract}

Keywords_-game-based learning, technology-enhanced learning, e-Adventure, ZeroCoupalge, mobile-game-based learning, context awareness, m-learning

\section{Introduction}

Mobile technologies have proved to have enormous potential in a variety of contexts, particularly in higher education. The above is attributed to the evolution of education as a result of technological advancements. According to statista, mobile device penetration has risen to $67 \%$ in recent years, growing by $2.4 \%$ from 2018 to 2019 [1]. Android's smartphone market share is predicted to hit $85.5 \%$ in 2022 . The most popular mobile operating system is Android with $85.41 \%$ of users, followed by Apple iOS with $13.2 \%$ of users and other operating systems with $1.3 \%$ of users cite WinNT. The use of mobile devices and technology to promote, assist, expand, and broaden the scope of teaching and learning is known as mobile learning [2]. Quinn defines m-learning as training which occurs with the assistance of mobile devices. In 
accordance with this definition, several authors consider the mobile device as a tool ubiquitaire which makes it possible to combine training, work, and entertainment in significant ways [3]. E-learning is described by Pinkwart as learning that is supported by digital electronic resources, and m-learning as learning that is supported by mobile and wireless devices [4]. Learning-oriented methodologies can be developed to enable students to engage in continuous learning processes using any computer, anywhere, and at any time. Students, for example, may use computers or mobile devices to navigate educational sites without having to be in a physical classroom [1]. M-learning is the most recent form of learning in which new learning techniques are delivered by mobile devices and applications [5]. According to Berking and al. (2013), the cell phone's unique features, such as communication, portability, GPS, and cameras, have great potential to enhance the teaching and learning experience [6]. On the other hand, Game Based Learning is gaining a lot of attention from researchers and practitioners because it combines learning and fun. Furthermore, multiple studies have shown that educational games can improve students' earning outcomes and inspire them to learn [7]. According to Prensky (2005) [8], previous motivating factors are ineffective for today's generation of learners. As a result, learning methods and media have evolved over time to deliver information. Learners would be more likely to learn if the material were presented in an engaging manner. This adaptation began with traditional learning and progressed to e-learning systems and then to educational video games. In an educational sense, the integration of mobile learning into gaming has been increasing. The word "Mobile Game Based Learning" (MGBL) refers to the creation of mobile educational games that combine mobile learning (m-learning) with applications on mobile phones (Chung, Hwang, \& Lai, 2019) [9]. Mobile game-based learning is a simulation, which provides an overview of the lesson's theme, is designed for various stages of education, is personalized for the new curriculum, and can be played on a mobile device [10]. However, only a small amount of research has been done on how to develop Mobile Game Based learning for students and what design features should be considered for a stronger educational result. Moreover, the mobile aircraft equipped with additional sensors, such as receivers, numerical GPS and cameras, help to acquire contextual information about training such as the site, activity and connectivity. That helps to improve and assist the activities of training. Context awareness is the ability to recognize any information that is required to define an individual (person, place, or object that can be integrated into a given environment) [11]. Mobile Game Based Learning, which takes into account the context of training, is called context awareness Mobile Game Based Learning. In this paper, we propose a new architecture for Game Based learning. Authoring tool based on contextualization and using the zeroCouplage Framework. This architecture will automatically and dynamically meet the needs of the user and provide him with appropriate Mobile Game Based Learning according to the context changes. 


\section{Related work}

\subsection{Adaptive context-aware mobile game based learning}

Before we can talk about adaptive and context-aware Mobile Game Based Learning, we need to understand what context based adaptation is in general and Mobile Game Based learning adaptation in particular. "Context" is described as "a piece of knowledge that can be used to describe the circumstance of a person in an interaction." (Abowd et al. 1999) [12]. Furthermore, Dey et al. (1999) described context, as the user's spatial, social, mental, or informative state. Pascoe (1998) proposed a similar definition, believing that context is a set of physical and conceptual factors related to a particularity [13]. Place, orientation, date, time, surrounding people and things, emotional condition, identity, season, and temperature have all been used by other researchers to explain the current state of any consumer (Ryan et al. 1997; Brown and al. 1997; Dey1998) [14]. These meanings and examples lead to the principle of context-awareness, which is described by Abowd and al. (1999) as follows: "A machine is context aware if it uses context to offer specific knowledge and/or resources to the user, where relevancy is determined by the user's task." Furthermore, several scholars believe that (Ryan and al. 1997; Fickas and al.1997; Pascoe1998; Brown 1998) context-aware application provides information, conducts tasks, tracks updates, implements programs, interprets scenarios, responds to users' needs in a flexible manner, and takes into account the contexts defined by the system sensors.

\subsection{Mobile devices}

Context-based learning strategies are made possible by mobile devices. Learners can play and learn in an atmosphere close to that of the game using this approach. Learners, for example, use their handheld devices to learn about Taiwanese society (e.g., religion) by playing a mobile educational game in temples and churches (Chen, Shih, \& Ma, 2014) [15]. A mobile device is a portable computing device, which often has a display and an input/ output interface, with necessary interaction devices or accessories. It represents the primary learning tool through which the learner can obtain learning materials and stay in touch with their instructor and peers. The capabilities of mobile devices such as processing power, memory, storage, and other performance benchmarks affect the size and type of learning material provided such as its degree of complexity, size, and quality. These abilities have a direct effect on the speed and simplicity of the interaction between the learner and the learning material [16]. Popular mobile devices can be classified according to their characteristics as below:

- Tablet PC: Small size laptops like, UMPC (Ultra Mobile PC), laptops, netbooks, etc. These devices are equipped with wireless network connections like WiFi, Bluetooth, 3G / 4G, etc. Their processor power, screen resolution, and system memory are sufficient to use most multimedia content. The only downside is that 
their mobility is less important than that of a PDA and a mobile phone, but at the same time, more than a laptop [17].

- PDA: It has a small size but significant processor power. It can recognize handwriting and can do many tasks. Normally, it has a larger screen than a smartphone.

- Mobile phone: Mobile phones can be used for voice communication and for sending SMS (Short Message Service) text messages. Their computing power and transfer speeds are low. But with the commercialization of $3 \mathrm{G} / 4 \mathrm{G}$, mobile phones have the possibility of accessing the Internet via WAP (Wireless Application Protocol), GPRS (General Packet Radio Service) or 3G / 3G, etc.

- Smartphone: Smartphones combine the capabilities of PDAs and mobile phones. They have the most modern interaction techniques, like the multi-touch screen. Processor power and memory are also growing steadily. Several types of operating systems are used, like Windows mobile, Windows CE, Symbian, Linux mobile, Palm, Android, iOS, etc. Other devices, such as personal multimedia players (MP3, MP4, etc.) or portable game consoles (Sony PSP, Nintendo DS) are also considered as mobile devices, because they have the ability to read multimedia files and have sufficient memory.

\subsection{ZeroCouplage framework}

The Framework Zerocouplage proposed by (Ettifouri, E.H., Rhouati, 2015) can develop different types of applications with a single layer of business. Hence the name Zerocouplage, signifying zero coupling between the presentation layer and the business layer. ZeroCouplage meets the major needs of the development of an effective LG, which are: reusability, maintainability and cost reduction. The game designer does not need any specific skills in JAVA/JEE or Android to develop Multiplatform Game-Based Learning on both web and mobile platforms. ZeroCouplage is based on the design pattern M2VC; a derivative of the MVC Pattern. M2VC Design Pattern Structure: The Model-VirtualView-Controller (M2VC) design pattern is a derivative of the Model-View-Controller (MVC) design pattern. It is intended for the development of applications with multi-support, such as web, mobile, and desktop support. The M2VC and MVC share the same concept of the model, but the M2VC controller uses a virtual view to manipulate the concrete views. It loads the concrete view according to the selected support. (See Figure1). 




Fig. 1. M2VC design pattern structure

\section{Problematic}

Authoring software for Mobile Learning Games (MLGs) makes it easy for teachers to create and use apps that are adapted to their individual needs and to the context [17] [16]. Mobile Game Based Learning is particularly popular among teachers for educational tours and field trips. MLGs, in reality, have all of the ingredients to pique students' interest and involve them in learning experiences [18] [19], [20]: Competition, prizes, and experimentation are all game mechanics, as are positioned learning in real-world environments and the physical work required to get to the right location. In recent times, numerous Mobile Game Based Learning authoring tools have been suggested. For researchers, the creation, production, and administration of such authoring resources are arguably difficult and time-consuming activities. The issue of developing game-based learning for different platforms is much clearer. This is a significant burden for both mobile game makers, who must adapt their games for each of these platforms, and beginner educators, who need computer skills. LG designers must stay up with this quick growth and offer their products and services on a variety of platforms in order to reach as many people as possible, particularly the younger generation. Game-based learning, on the other hand, must deal with large development expenditures as well as the problem of all. To face the problem of multi-platform targeted applications, the ZeroCouplage Framework was introduced in this article as a solution for Mobile Game Based Learning Designers. The main aim of this framework is to code once and choose the target platform without injecting new code into the existing application nor having knowledge of the programming languages for Web, mobile, or desktop since it is based only on the language Java. 


\section{Our contribution: The proposed eAdventure architecture using ZeroCouplage framework}

\subsection{The existing eAdventure, and its limitations}

The eAdventure platform is a free and open source platform for creating instructional and learning games. It facilitates novice users' adoption and brings them closer to the game by simplifying the process of building 2D point \& click conversational adventure games.eAdventure began in 2007, as a research project of the "e-UCM" laboratory and as the major result of Pablo Moreno's doctoral thesis. Many individuals have contributed to eAdventure until it has become a reliable product with over 10,000 downloads per year (source: Source-Forge.net) and is available in ten languages. The eAdventure Editor and the eAdventure Engine Core are the two main components of the current eAdventure architecture. The eAdventure Editor (Game description) enables a beginner designer to select various game components such as characters, items, assets, and game scenarios [21]. Based on the aforementioned criteria, we may conclude that the existing eAdventure design is severely limited in terms of functionality. For this reason, we propose a new architecture for the eAdventure Game Based Learning Authoring tool based on contextualization and utilizing the zeroCouplage Framework. This new architecture will adapt to the user's demands automatically and dynamically, providing him with relevant Mobile Game Based Learning as the situation evolves.

\subsection{The proposed architecture of eAdventure using ZeroCouplage framework}

The current eAdventure architecture works with the MVC design pattern (model, view, and controller) that ensures a separation between the layers. However, these multiple views offered by the MVC are just at the level of a single platform. Regarding the "multiplatform adaption" design principle: The proposed authoring tool architecture eAdventure uses the framework ZeroCouplage. The idea of integrating the ZeroCouplage framework is to use the same codebase (XML) edited by the eAdventure editor. The eAdventure engine could generate Context Awareness Mobile Game Based Learning for multiple platforms meeting the criteria already set by the designer (See Figure 2). The three target platforms are (web, desktop, mobile) represented respectively by those technologies: html/css/js, JavaFx and Android for mobile (See Figure 3).These platforms have the same business logic and work in the same way in the backend. The difference arose at the level of the presentation that is represented by the views. Indeed, for web presentation, we use jsp or html pages, for desktop we use the scenes of JavaFx and for mobile, we have activities. 


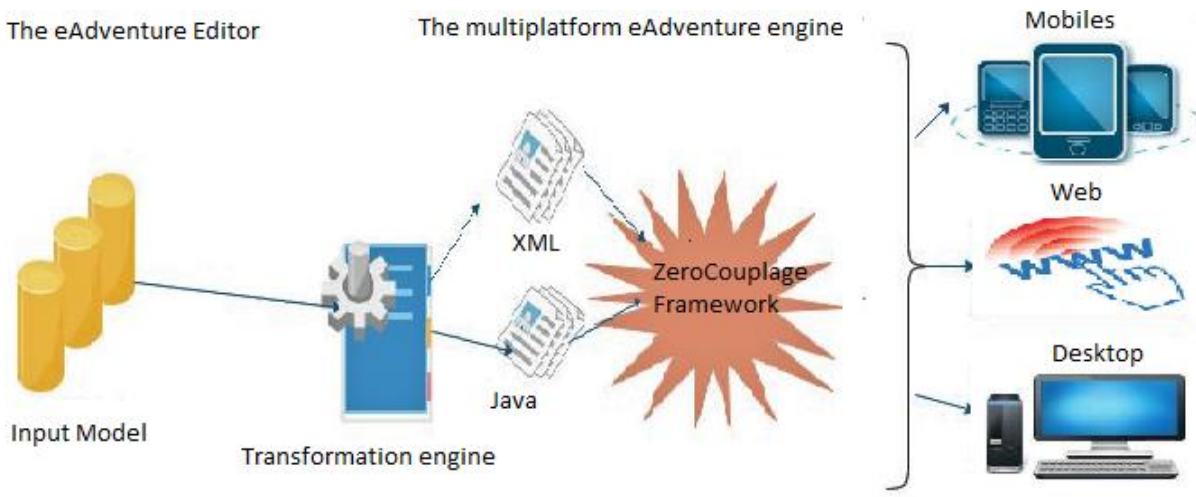

Fig. 2. The proposed architecture of eAdventure using ZeroCouplage framework



Fig. 3. The game based learning platforms and technologies (web, mobile, desktop)

\section{Conclusion}

In this work we have introduced the new eAdventure architecture, which will enable the creation of multiplatform context-aware mobile games-based learning. The key benefit of this method is that eAdventure can readily accept extensions to welcome a variety of mobile device platforms. Our future projects include the creation and evaluation of accessible context-aware Mobile Game-Based Learning using the proposed new eAdventure architecture. The next step will be the adaptation of context-aware Mobile Game Based Learning to different learner players' profiles, which includes cognitive abilities, learning styles (figurative, symbolic, and semantic), and these methods will allow us to tailor the generated MGBL to their preferences and level, resulting in an effective learning experience. 


\section{References}

[1] Paola Vallejo-Correa, Julián Monsalve-Pulido, and Marta Tabares Betancur. A systematic mapping review of context-aware analysis and its approach to mobile learning and ubiquitous learning processes. Computer Science Review, 39:100335, 2021. https://doi.org/ 10.1016/j.cosrev.2020.100335

[2] Masoud Hashemi, Masoud Azizinezhad, Vahid Najafi, and Ali Jamali Nesari. Retracted: What is mobile learning? Challenges and capabilities, 2011. https://doi.org/10.1016/j. $\underline{\text { sbspro.2011.10.483 }}$

[3] Clark Quinn. mlearning: Mobile, wireless, in-your-pocket learning. LiNE Zine, 2006:1-2, 2000.

[4] Niels Pinkwart, Heinz Ulrich Hoppe, Marcelo Milrad, and Juan Perez. Educational scenarios for cooperative use of personal digital assistants. Journal of computer assisted learning, 19(3):383-391, 2003. https://doi.org/10.1046/j.0266-4909.2003.00039.x

[5] H Seyed Ebrahim, K Ezzadeen, and Alhazmi AK. Acquiring knowledge through mobile applications. International Journal of Interactive Mobile Technology, 9(3):71-74, 2015.

[6] Peter Berking, Marcus Birtwhistle, Shane Gallagher, and J Haag. Mobile learning survey report. Advanced distributed learning initiative, 2013.

[7] Ahmed Tlili, Fathi Essalmi, Mohamed Jemni, Nian-Shing Chen, Ronghuai Huang, Daniel Burgos, et al. The evolution of educational game designs from computers to mobile devices: A comprehensive review. Radical Solutions and eLearning, pages 81-99, 2020. https:// doi.org/10.1007/978-981-15-4952-6_6

[8] Marc Prensky. J computer games and learn| ng: D $1 \mathrm{~g}$ ital game-based learn $1 \mathrm{ng} .2005$.

[9] Ching-jung Chung, Gwo-jen Hwang, and Chiu-lin Lai. A review of experimental mobile learning research in 2010-2016 based on the activity theory framework. Computers \& education, 129:1-13, 2019. https://doi.org/10.1016/j.compedu.2018.10.010

[10] Ucu Cahyana, Maria Paristiowati, Dwi Amelia Savitri, and Siti Nuryana Hasyrin. Developing and application of mobile game based learning (mgbl) for high school students performance in chemistry. Eurasia Journal of Mathematics, Science and Technology Education, 13(10):7037-7047, 2017. https://doi.org/10.12973/ejmste/78728

[11] Sélinde van Engelenburg, Marijn Janssen, and Bram Klievink. Designing context-aware systems: A method for understanding and analysing context in practice. Journal of logical and algebraic methods in programming, 103:79-104, 2019. https://doi.org/10.1016/ j.jlamp.2018.11.003

[12] Anind K Dey, Daniel Salber, Masayasu Futakawa, and Gregory D Abowd. An architecture to support context-aware applications. Technical report, Georgia Institute of Technology, 1999.

[13] Jason Pascoe. Adding generic contextual capabilities to wearable computers. In Digest of papers. second international symposium on wearable computers (cat. no. 98ex215), pages 92-99. IEEE, 1998. https://doi.org/10.1109/iswc.1998.729534

[14] Nick S Ryan, Jason Pascoe, and David R Morse. Enhanced reality fieldwork: the contextaware archaeological assistant. In Computer applications in archaeology. Tempus Reparatum, 1998.

[15] Cheng-Ping Chen, Ju-Ling Shih, and Yi-Chun Ma. Using instructional pervasive game for school children's cultural learning. J. Educ. Technol. Soc., 17(2):169-182, 2014.

[16] Lamyae Bennis and Said Amali. From learning game to adaptive ubiquitous game based learning. International Journal of Emerging Technologies in Learning, 14(16), 2019. $\underline{\text { https://doi.org/10.3991/ijet.v14i16.10701 }}$ 
[17] Lamyae Bennis and Said Benhlima. Toward a new approach: extending a game-based learning authoring tool eadventure to multiple mobile devices. In Europe and MENA Cooperation Advances in Information and Communication Technologies, pages 47-56. Springer, 2017. https://doi.org/10.1007/978-3-319-46568-5_5

[18] Iza Marfisi-Schottman and Sébastien George. Supporting teachers to design and use mobile collaborative learning games. In International Conference on Mobile Learning, pages $3-10,2014$.

[19] Nadia Bianchi-Berthouze. Understanding the role of body movement in player engagement. Human-Computer Interaction, 28(1):40-75, 2013.

[20] Aous Karoui, Iza Marfisi-Schottman, and Sébastien George. Jem inventor: a mobile learning game authoring tool based on a nested design approach. Interactive Learning Environments, pages 1-28, 2020. https://doi.org/10.1080/10494820.2020.1753214

[21] Lamyae Bennis, Said Amali, et al. Generating adaptive learning games to multiple mobile platforms. In International Conference on Advanced Intelligent Systems for Sustainable Development, pages 129-135. Springer, 2019. https://doi.org/10.1007/978-3-030-36653$\underline{7} 13$

\section{$7 \quad$ Authors}

Lamyae Bennis received the Ph.D. degree in computer sciences from Moulay Ismail University, Meknes, Morocco, in 2020. She is currently a member of the Mathematics and Computer Sciences Laboratory, Faculty of Sciences of Meknes. Her research interests include educational technologies, intelligent systems, learning analytics, and serious games. Her current project is the conception and development of an authoring tool in order to generate adaptive ubiquitous learning games.

khalid kandali was born in Casablanca, Morocco, November 26, 1985. He received the master's degree in network and computer science from the Faculty of Sciences and Techniques, University of Hassan 1st, Settat, Morocco. He is currently pursuing the Ph.D. degree in computer science with the Graduate School of Technology, Moulay Ismail University, and Meknes, Morocco. He is also involved in the vehicular ad hoc networks, wireless sensor networks, and machine learning.

Hamid Bennis was born in Meknes, Morocco, in September 1977. He received the $\mathrm{Ph} . \mathrm{D}$. degree in computer science and telecommunications from Mohammed V University at Agdal, Rabat, Morocco, in 2011. He is currently a Professor of computer science and telecommunications with the Department of Computer Science, Graduate School of Technology, Moulay Ismail University, and Meknes. He is also the Head of the research team Communication Systems, Artificial Intelligence, and Mathematics. $\mathrm{He}$ is also a contributing author of a number of refereed journals, book chapters, and proceeding articles in the areas of wireless communications. His current research interests include mobile ad hoc networks; wireless sensor networks, wireless power transmission (WPT), machine learning, and microwave hybrid circuits.

Article submitted 2021-08-02. Resubmitted 2021-10-18. Final acceptance 2021-10-18. Final version published as submitted by the authors. 